\title{
Moderne Ökonomie ohne Wachstumszwang: ein Wunschtraum?
}

Eine kapitalistische Wirtschaft „muss“ wachsen, aber wo kommt dieser Wachstumsimperativ her? Und wie ist zu erklären, dass gerade die entwickelten kapitalistischen Länder immer weniger in der Lage sind, dem selbst gesetzten Wachstumsimperativ gerecht zu werden? Konventionelle ökonomische Wachstumstheorien sind nicht in der Lage, diese Fragen befriedigend zu beantworten. In dem Beitrag wird ein alternativer soziologischer Ansatz skizziert, der neue Antworten auf die Frage bietet, warum die Bäume kapitalistischer Dynamik nicht in den Himmel wachsen.

CHRISTOPH DEUTSCHMANN

\section{1. „Wachstumszwang" und „Wachstum“ - erste Annäherungen}

Im Titel dieses Aufsatzes steht der schwierige und erläuterungsbedürftige Begriff „Wachstumszwang“. Es geht also nicht etwa um eine Ökonomie ohne Wachstum. Diese ist in den entwickelten Industrieländern (EU, USA, Japan) heute schon weitgehend Realität; nur in Schwellenländern wie China, Indien und Brasilien, zunehmend auch in Afrika, ist noch hohes Wirtschaftswachstum festzustellen. Das NullWachstum, wie es heute von grünen und auch konservativen Theoretikern mit primär umweltpolitischen Begründungen gefordert wird, ist keine Utopie, sondern Wirklichkeit, allerdings keine erfreuliche. Die gesamte Gesellschaft leidet darunter, denn diese neue Realität bedeutet Beschäftigungsabbau, Arbeitslosigkeit, staatliche Finanzierungsengpässe, wirtschaftlichen Niedergang, individuelle Perspektivlosigkeit, soziale Konflikte.

Warum ist das so? Warum „muss" die Wirtschaft wachsen? Der Begriff „Ökonomie“ ist bekanntlich aus dem griechischen Wort für Haushalt bzw. Haushaltslehre abgeleitet. Ein Haushalt „muss“ nicht wachsen; es kann zwar sein, dass seine Ausgaben steigen, wenn die Zahl der Haushaltsmitglieder zunimmt oder ihre Bedürfnisse sich verändern. Aber es ist auch kein Problem, wenn die Ausgaben und Einnahmen gleich bleiben. Wenn weniger Personen im Haushalt leben, oder die Leute bescheidener leben wollen, können die Ausgaben auch zurückgehen, ohne dass dies nachteilige Folgen für irgendjemand hätte. Warum kann das Wirtschaftssystem als Ganzes es nicht genauso machen? Vorläufig ist nur festzustellen: Das System verhält sich entgegen der irreführenden Bezeichnung als „Ökonomie“ eben nicht wie ein Haushalt, sondern wie ein Fahrrad: Wenn es nicht ständig weiterfährt, fällt es um. Das sogenannte „Wachstum“ ist nicht einfach mit höherem „Wohlstand“ gleichzusetzen. Die Wirtschaft „wächst“ auch, wenn mehr Unfallschäden oder Umweltverwüstungen repariert werden müssen. Und selbst in vielen Jahren, in denen die Statistiker noch ein ,reales “Wachstum festgestellt haben, sind die realen Masseneinkommen in Deutschland und in anderen entwickelten Industrieländern nicht mehr gestiegen oder sogar zurückgegangen. Wachstum scheint vielmehr eine Bedingung dafür zu sein, dass wir unseren gegebenen Lebensstandard auch nur halten können. Daraus ergibt sich der „Wachstumszwang“, von dem hier die Rede sein soll.

Über die Ursachen dieses Zwangs gibt es eine breite Diskussion, deren Schwierigkeiten aber immer noch oft unterschätzt werden. Ein Grundproblem ist, dass es gar nicht möglich ist, Wachstum genau zu definieren. Wachstum bedeutet ja nicht einfach eine größere Menge von Gütern des gleichen Warenkorbes, sondern Entwicklung, d.h. Erfindung und Vermarktung neuer Güter, Dienstleistungen, Produktionsmittel, Organisations- und Produktionskonzepte. Schon für die Güter eines gegebenen Warenkorbes (z.B. Schweinefleisch, Autoreifen, Therapiestunden, Elektrizität) gibt es kein gemeinsames Maß; erst recht nicht 
messbar ist das ständig entstehende Neue. Das Einzige, was die wirtschaftliche Produktion und überdies die Nachfrage nach ihr in ihrer unvorstellbaren Heterogenität in Beziehung zueinander setzen kann, ist Geld; nur Geld hat die rätselhafte Fähigkeit, Unvergleichbares vergleichbar zu machen. Der Wachstumszwang ist also im Kern ein Zwang zur Geldvermehrung; nur in diesem Sinn ist er quantitativ. Aber was sich in der Zunahme der Wertsumme des Geldes ausdrückt, wissen wir nicht, und wir wüssten es selbst dann nicht, wenn es gelänge, eine inflationäre und eine „reale“ Wertvermehrung zuverlässig voneinander zu unterscheiden. Denn was ist eine „reale“ Wertvermehrung?

\section{Wachstum ist nicht nur ökonomisch, sondern auch soziologisch zu erklären}

Ich werde diese Probleme, über die sich endlos grübeln ließe, hier nicht weiterverfolgen. Viele Äußerungen zu der Diskussion um Wachstum und Wachstumskrisen klingen so, als ob es den Wachstumszwang gar nicht gäbe, als ob es eine rein politische Entscheidung wäre, ob die Wirtschaft wachsen soll oder nicht. Manche scheinen zu glauben, dass die Wirtschaft mit einem Bedienungsknopf ausgestattet ist, und wenn wir diesen Knopf einmal drücken, kommt das Wachstum, wenn aber zweimal, dann hört es auf. Und wenn wir nach links drehen, kommt qualitatives Wachstum heraus, nach rechts quantitatives. So scheint die Frage nur noch zu sein: Wie oft wollen wir den Knopf drücken, wohin wollen wir ihn drehen? Doch leider gibt es den Knopf nicht. Die Wirtschaftstheorie und -politik behaupten zwar, dass sie ihn haben, aber auch hier sind sich die Experten nicht einig: Die einen schwören auf keynesianische Nachfragesteuerung, die anderen auf sogenannte „Angebotspolitik“, wiederum andere sagen, dass staatliche Interventionen gleich welcher Art vom Übel sind und dass es nur auf den richtigen institutionellen Rahmen ankomme. Ich werde auch diese Kontroversen hier einfach beiseite lassen und gehe von folgender These aus: Wachstum geht auf emergente gesellschaftliche Prozesse zurück, die politisch kaum steuerbar sind und im Kern gesellschaftlich erklärt werden müssen. Ich werde deshalb auch nicht auf die Frage eingehen, ob und welche Art von Wirtschaftswachstum wünschenswert ist. So wichtig die Frage ist: Um sie mit Aussicht auf praktische Konsequenzen zu diskutieren, sollten wir erst einmal verstanden haben, wie das Wachstum kapitalistischer Wirtschaften überhaupt funktioniert. Dazu reichen rein ökonomische Modelle nicht aus, denn Wachstum ist etwas, was alle Bereiche der Gesellschaft einbezieht und deshalb auch soziologisch erklärt werden muss. Erst auf der Basis einer so gewonnenen, hoffentlich tragfähigen Diagnose haben wir eine Chance, praktisch folgenreiche normative Empfehlungen zu entwickeln.

Was ist eine „soziologische“ Erklärung? Das wird vielleicht am leichtesten deutlich, wenn ich zunächst an einigen
Beispielen erläutere, was sie nicht ist. In der aktuellen Wachstums- und Konsumdebatte sind Autoren einflussreich, die den Wachstumszwang vor allem als Problem einer durch Wachstumsideologien fehlgeleiteten individuellen Motivation deuten. Die Menschen seien von einem "Mehrgott" besessen, behauptet Peter Gross (1994). Meinhard Miegel (2010) glaubt, die Konsumenten hingen wie Drogensüchtige an der Nadel des Wirtschaftswachstums und fielen in Panik, wenn der Nachschub nachlässt. Was immer man von diesen Thesen halten mag, um soziologische Erklärungen handelt es sich jedenfalls nicht. Denn erstens lassen sich Kollektivphänomene wie wirtschaftliches Wachstum nicht direkt aus individuellen Motiven ableiten. Zweitens sind die erwähnten Aussagen in ihrer generalisierenden Zuspitzung auch empirisch falsch. Zwar gibt es Erscheinungen wie Konsumgier und Kaufrausch; es gibt auch die Geldgier bei Vermögenden, die trotz oder gerade wegen ihres Reichtums nie genug haben können. Aber der Wunsch von Geringverdienern nach einem höheren Einkommen kann mit dieser Art von Gier nicht einfach gleichgesetzt werden. Und nicht alle Menschen „wollen“ das Wachstum, nicht alle sind begierig darauf, noch mehr zu konsumieren oder noch höhere Renditen auf ihre Vermögen zu kassieren. Viele sind im Grunde zufrieden; sie haben die beständige Belästigung durch die Werbung satt und möchten gern in der gewohnten Weise weiterleben, wenn sie es sich nur leisten können.

Eine andere, vor allem unter Ökonomen beliebte These lautet, bei dem Wachstum handele es sich um eine in der Logik des Marktes, der Evolution, der Kapitalverwertung (oder durch was auch immer) begründete objektive Gesetzmäßigkeit. Diese entfaltet sich über unsere Köpfe hinweg, ob wir es wollen oder nicht. Auch das ist keine soziologische Erklärung, denn gesellschaftliche Prozesse und Aggregatphänomene setzen sich niemals rein „objektiv“ durch wie Naturgesetze, sondern immer durch die Vermittlung individuellen Handelns. Handeln aber wird nicht einfach von externen Faktoren determiniert, sondern verfügt über wie auch immer begrenzte Freiheitsspielräume, die seine vollständige Berechenbarkeit ausschließen. Überdies handelt es sich bei der erwähnten Aussage wiederum um eine empirisch falsche Generalisierung. Denn die behauptete „Gesetzmäßigkeit“ des Wirtschaftswachstums stimmt mit den historischen Tatsachen keineswegs überein. Im Gegenteil: Nicht Wachstum, sondern stationäre Reproduktion war bis vor etwa 200 Jahren der historische Normalzustand, wie wir noch genauer sehen werden. Und obwohl es in den beiden letzten Jahrhunderten in der Tat einen starken Wachstumstrend gegeben hat, war dieser Trend doch immer wieder durch schwere Krisen unterbrochen, wie wir gerade in der Gegenwart wieder erleben.

Der Schlüssel zur Erklärung des Wachstums liegt weder in den gesellschaftlichen Strukturen, noch in den individuellen Motiven allein, sondern in der Verbindung beider. Wenn viele Menschen das Gleiche tun, hat ihr Handeln oft kollektive Ergebnisse, die den Absichten der Handelnden zuwiderlaufen. Viele möchten z.B. gern zum aktuellen 
Schnäppchenpreis Benzin tanken, aber genau die Tatsache, dass so viele zur Tankstelle eilen, treibt den Preis in die Höhe und durchkreuzt die ursprüngliche individuelle Absicht. Was uns als „gesellschaftlicher Zwang“ erscheint, ist häufig nichts anderes als die Folge einer solchen ungeplanten Verkettung individueller Handlungen. Wir müssen also bei der Erklärung des Wachstumsphänomens sorgfältig zwischen der Ebene der Handlungen einerseits und der der kollektiven Strukturen andererseits unterscheiden. Beide bedingen einander, lassen sich aber trotzdem nicht aufeinander reduzieren. Die Aufgabe einer soziologischen Erklärung liegt darin, die geplanten und ungeplanten Wechselbeziehungen zwischen beiden $\mathrm{zu}$ analysieren und zu zeigen, wie der Wachstumszwang als Aggregateffekt individueller Handlungen entsteht (für eine formale Beschreibung dieses Vorgehens vgl. Esser 1993). Wie das gehen könnte, will ich skizzieren (ausführlicher dazu: Deutschmann 2009, 2011).

\section{Die Entgrenzung der Märkte als historische und sozialstrukturelle Voraussetzung}

Ich beginne mit der Ebene der makrosozialen Struktur. Festzustellen ist zunächst, dass es keineswegs zur „Natur“ der Wirtschaft gehört, wachsen zu müssen. Der historische „Normalzustand" der Wirtschaft, wie er über Jahrtausende bis vor rund 200 Jahren herrschte, war vielmehr, wie wir vor allem seit den Untersuchungen Maddisons (2001) wissen, die stationäre Reproduktion, also die schlichte Wiederherstellung des überkommenen ökonomischen Zustandes mit höchstens kleinen Veränderungen über lange Zeiträume. Erst seit dem frühen 19. Jahrhundert ist es, ausgehend von Europa und den USA und dann sukzessive in der restlichen Welt, zu einer wahren "Wachstumsexplosion“ der Wirtschaft gekommen. In dem nur relativ kurzen historischen Zeitraum von zwei Jahrhunderten wuchs die Weltwirtschaft um ein Vielfaches stärker als in der gesamten früheren Geschichte (Maddison 2001, S. 28, 265). Was ist damals geschehen? Üblicherweise fällt hier das Stichwort: industrielle Revolution. Aber das ist keine Erklärung, sondern nur eine andere Beschreibung des Phänomens der Wachstumsexplosion selbst. Die entscheidende Bedingung bestand, wie Karl Marx und später Karl Polanyi gezeigt haben, in etwas anderem: in der mit den bürgerlichen Reformen des späten 18. und frühen 19. Jahrhunderts durchgesetzten Entgrenzung der Märkte, in ihrer Ausdehnung von Gütern und Dienstleistungen auf den Boden, die hergestellten Produktionsmittel und vor allem die Arbeitskraft. Kapitalismus ist nicht einfach „Marktwirtschaft“, wie heute noch immer ungenau gesagt wird, denn Märkte hat es fast immer in der Geschichte gegeben. Er ist vielmehr entgrenzte Marktwirtschaft, in der nicht nur die Arbeitsprodukte, sondern auch die Voraussetzungen der Produktion
- Land, Produktionsmittel und vor allem die Arbeitskraft selbst zu Waren geworden sind. Der gesamte Kreislauf gesellschaftlicher Reproduktion wird durch die Warenform erfasst Neben Märkten für Produkte und Dienstleistungen gibt es nun Märkte für Land, produzierte Produktionsmittel, Arbeit, schließlich Kapital- und Finanzmärkte. Und neben dieser sachlichen Entgrenzung sind die Märkte in einem weiteren, räumlichen Sinn entgrenzt, indem sie einen weltumspannenden Charakter angenommen haben. Schon seit seinen historischen Anfängen stellte der Kapitalismus ein „Weltsystem“ (Wallerstein 1979) dar.

Die „große Transformation“, wie Polanyi diese zu Beginn des 19. Jahrhunderts begonnene Entgrenzung der Marktwirtschaft bezeichnet hat, hat die Voraussetzungen für die bis heute anhaltende Dynamik des Kapitalismus geschaffen. Es macht nämlich einen grundlegenden Unterschied aus, ob man für Geld nur Güter und Dienstleistungen kaufen kann oder aber die Bedingungen der Produktion. Die entscheidende Neuerung liegt vor allem in der Schaffung eines Marktes für freie Arbeit (die Betonung liegt auf frei, denn auch Sklavenmärkte hat es fast immer gegeben). Güter und auch Maschinen oder Computer sind Objekte mit bestimmbaren Eigenschaften und Leistungspotenzialen. Die freie, also an ihrer Vermarktung selbst interessierte Arbeitskraft dagegen ist ein offenes, mit kreativen Fähigkeiten ausgestattetes Potenzial, das genuin Neues hervorbringen kann. Diese Potenziale in organisierter Form auszuschöpfen, ist die Mission des kapitalistischen Unternehmers - einer sozialen Figur, die erst auf der Basis der modernen Entgrenzung der Märkte entstehen konnte.

Wenn ich den Begriff Kreativität verwende, denke ich nicht nur an die großen Erfindungen, auch nicht nur an die „Visionen“ des Unternehmers, sondern auch an die vielen „kleinen“, alltäglichen Ideen der Beschäftigten und Konsumenten, ohne die neue Techniken und Produkte nicht entstehen und funktionieren könnten. Die kreativen Fähigkeiten gesellschaftlicher Arbeit sind als Gesamtheit niemals abschließend bestimmbar. Erst recht nicht können sie als Gesamtheit - für wie viel Geld auch immer - privat angeeignet werden. Einlösbar ist der Eigentumsanspruch auf die lebendige Arbeit nur als nie zu vollendender Prozess, das heißt durch die Verwandlung des Geldes in Kapital und durch die kapitalistisch organisierte Verwertung der innovativen Potenziale der Arbeit. Der Widerspruch zwischen dem quantitativ fixierten Charakter jeder Geldsumme und der Unbestimmbarkeit der kreativen Potenziale der Arbeit kann nur dynamisch überwunden werden: nicht durch den einzelnen Gewinn, sondern durch die „rastlose Bewegung des Gewinnen““ (Marx 1988, S. 168). Neben die sachliche und die räumliche tritt somit die zeitliche Entgrenzung der Märkte.

In dieser dreifachen Entgrenzung wurzelt der Trieb des Kapitalismus zu immer neuen Erfindungen, zu technischen und industriellen Revolutionen, zu neuen Konsummoden, zu immer neuen Umwälzungen auch der Organisationsstrukturen und Institutionen. Das in Kapital verwandelte Geld hat sich als ein wahres „Sesam-Öffne-Dich“ erwiesen und Innovationen angestoßen, von denen man in früheren Zeit- 
altern nicht geträumt hätte. Kreativität und Innovation, nicht einfach nur höhere „Produktivität“" oder „Rationalisierung“ sind die Haupttriebkräfte kapitalistischer Entwicklung, wie die neuere Forschung (z.B. Freeman/Louca 2001) im Anschluss an Schumpeter betont. Kapitalistische Dynamik ist zwar, wie ebenfalls schon Schumpeter hervorgehoben hat, nicht nur kreativ, sondern auch destruktiv; sie bedeutet immer auch die Zerstörung gegebener ökonomisch-sozialer Strukturen, und sie ist auch ökologisch zerstörend. Aber alle diese Zerstörungen bilden nur die Kehrseite der globalen Zivilisation, die der moderne Kapitalismus hervorgebracht hat und die unser heutiges Leben prägt.

Der Wachstumszwang entsteht - soviel ist zunächst festzuhalten - aus der Unmöglichkeit, das durch den modernen Kapitalismus geschaffene private Eigentumsrecht auf das lebendige Arbeitsvermögen einzulösen. Er kommt also vor allem von der Produktion her, nicht nur von den Konsumenten, obwohl diese auch mitspielen müssen. Der Unternehmer als Käufer des privaten Arbeitsvermögens hat - bei Strafe des Untergangs - kaum eine andere Wahl, als aus dieser unerschöpflich scheinenden Ressource immer mehr und immer Neues herauszuholen. Auf der Ebene des Einzelunternehmens drückt sich dieser Wachstumsimperativ in einem Gewinnimperativ aus: Nur wenn die Produktion einen Gewinn verspricht, kann sie sich lohnen und findet überhaupt statt. Ohne Gewinnaussicht ist es besser, abzuwarten und das Geld einfach zu behalten. Der Kapitalismus als System hat folglich nur die Wahl zwischen Wachstum und Niedergang; er kann sich nicht auf gleichbleibendem Niveau reproduzieren. Der Gewinnimperativ ist schließlich gleichbedeutend mit einem Verschuldungsimperativ. Wenn nämlich Unternehmen die von ihnen produzierten Waren mit Gewinn verkaufen wollen, dann setzen sie dabei eine Nachfrage voraus, die höher ist als die, die sie selbst mit ihren eigenen Kostenzahlungen geschaffen haben. Der Mehrwert kann nur dann realisiert werden - auf dieses Problem hat übrigens als erste Rosa Luxemburg aufmerksam gemacht -, wenn eine Zusatznachfrage auf den Plan tritt, die von außen kommen muss und durch zusätzlichen Kredit finanziert werden muss. Anders gesagt: Damit die einen Gewinne machen können, muss es andere geben (Haushalte, Unternehmen, Staaten), die sich verschulden und damit für die nötige Zusatznachfrage sorgen. Natürlich treibt dies das Wachstum zusätzlich an, denn die Schuldner müssen ja ihrerseits hart arbeiten, um das aufgenommene Kapital zu verwerten und mit Zins und Zinseszins zurückzuzahlen - mit der Folge, dass dann noch mehr Kredite nötig werden, um den Absturz zu vermeiden und so immer weiter.

\section{Mikro-Bedingungen wirtschaftlicher Wachstumszwänge}

Der Wachstumszwang und die daraus folgenden Gewinn- und Verschuldungszwänge ergeben sich letztlich aus der Entgren- zung des Marktes, aus der Tatsache, dass Arbeitskraft und Produktionsmittel unabhängig voneinander als „Lohnarbeit“ und „Kapital“ auf den Markt kommen: hier hatte Karl Marx zweifellos Recht. Aber das reicht als Erklärung des Wachstumszwangs noch nicht aus, denn es sind damit nur Bedingungen der Möglichkeit des Wachstums, noch keine zureichenden Ursachen genannt. Wachstum, so hatten wir gesehen, geht letztlich auf Innovation zurück. Innovation aber passiert nicht von allein; sie stellt sich auch nicht schon dadurch ein, dass Kapital zur Verfügung gestellt wird oder dass wissenschaftliche Erfindungen gemacht werden. Innovation ist, wie Schumpeter betonte, mehr als bloß „Invention“, d.h. Erfindung. Innovationen fordern einen Menschentypus, der nicht nur Ideen und Einfälle hat, sondern auch fähig und interessiert ist, sie zu marktfähigen Produkten weiterzuentwickeln. Wie ist die Entstehung dieses Menschentyps historisch und gesellschaftlich zu erklären, welches sind seine Motive?

Auch hier liefern Marx und Schumpeter zunächst die entscheidenden Hinweise: Die Entgrenzung der Märkte bedeutet - so lautet Marx' bekanntes Argument - die Teilung der Gesellschaft in zwei Klassen: die Klasse der Besitzer von Produktionsmitteln (inklusive Land) einerseits, die der Besitzer von Arbeitskraft andererseits. Allerdings ist die kapitalistische Klassenstruktur nicht so fest gefügt, wie Marx es darstellt. Denn anders als in allen historisch älteren Klassengesellschaften wird die individuelle Zugehörigkeit zu den beiden Hauptklassen im modernen Kapitalismus nicht durch den ständischen oder ethnischen Geburtsstatus vorherbestimmt, sondern ergibt sich allein aus dem puren Faktum des Besitzes oder Nichtbesitzes von Produktionsmitteln. Das heißt, die Klassenstruktur ist zwar kollektiv, nicht aber individuell geschlossen. Arbeiter oder kleine Selbstständige können zu Kapitalbesitzern aufsteigen, zumindest sich Hoffnungen auf einen solchen Aufstieg machen; umgekehrt können Kapitalbesitzer in die Arbeiterklasse absteigen. Die viel diskutierte „Unübersichtlichkeit“ kapitalistischer Klassenstrukturen (ein klassisches Thema der Soziologie) hängt mit dem widersprüchlichen, sowohl geschlossenen wie offenen Charakter dieser Strukturen zusammen. Objektiv wie subjektiv eindeutig sind nur die Positionen an den positiven und negativen Extremen der Klassendichotomie, d.h. der Besitzer großer Vermögen einerseits, der prekär situierten unteren Schichten der Arbeiterschaft andererseits. Zwischen den Extremen finden sich mehr oder weniger breite „Mittelschichten“, deren Mitglieder überwiegend keine feste Position einnehmen, sondern sich sozial in Bewegung befinden: Sie streben entweder „nach oben“ oder sie kämpfen gegen einen drohenden Abstieg. Marx hatte noch daran geglaubt, dass die Klassenstruktur sich im Verlauf der kapitalistischen Entwicklung in Richtung wachsender Eindeutigkeit verändern werde. Am Ende werde eine winzige Minderheit von Kapitalbesitzern einer Masse verelendeter und entrechteter Proletarier gegenüberstehen. Bekanntlich ist es so nicht gekommen. Die „Mitte“ ist nicht verschwunden, im Gegenteil scheint sie das gesellschaftliche Leben zumindest an der Oberfläche mehr denn je zu dominieren. 
Soziologisch lässt sich die innovative Dynamik des Kapitalismus zu einem großen Teil durch das Spannungsfeld zwischen den Extremen der Klassenstruktur und die dadurch ausgelösten Auf- und Abstiegsprozesse in der gesellschaftlichen „Mitte“ erklären. Innovation stellt, wie der amerikanische Soziologe Robert Merton (Merton 1968, S. 185f.) gezeigt hat, das charakteristische Verhalten von Menschen dar, die sich in einer widersprüchlichen sozialen Lage befinden. „Widersprüchlich“ ist die Lage der Mittelschichten in dem Sinne, dass viele Menschen Ambitionen verfolgen, die sich mit ihren meist nur bescheidenen Ressourcen (finanzielle Mittel, Bildungstitel, soziale Netzwerke) nicht realisieren lassen. Die Vermögenslosen befinden sich gleichsam in einer „double-bind“-Situation: Auf der einen Seite erscheint ihnen die Kluft, die sie von der Welt der Reichen trennt, unüberwindbar; auf der anderen Seite wird ihnen suggeriert, dass sie es schaffen können, wenn sie sich nur anstrengen.

Wichtig ist nicht die Kluft zwischen Zielen und Mitteln allein, sondern auch, wie sie von den Akteuren selbst wahrgenommen wird: Wird sie als unüberwindbar erlebt, kann das zu einer Haltung der Resignation oder Abkapselung führen, wie sie in den unteren Randschichten weit verbreitet ist, oder auch zu kämpferischer, organisierter Selbstbehauptung. Aber die Menschen in den Mittelschichten neigen viel eher dazu, die Kluft zwischen Arm und Reich als individuell überwindbar zu betrachten und suchen dann nach unkonventionellen Wegen zur Beschaffung der nötigen Mittel: Das ist es, was Merton als „Innovation“ bezeichnet. Es geht dabei allerdings keineswegs nur um „abweichendes Verhalten“ im Sinne von Kriminalität, wie Merton es primär im Auge hat. Diese Art von Innovation spielt zwar auch eine wichtige Rolle. Aber entscheidend ist, dass kapitalistische Gesellschaften einen (im Prinzip) legalen Weg des sozialen Aufstiegs durch Innovation eröffnen, den „unternehmerischen“ Aufstieg durch Erfolg am Markt - das ist Schumpeters bekannte These. Unternehmer sind Akteure, die (manchmal durchaus außerhalb oder am Rande der Legalität) ökonomische Routinen durchbrechen, neue technische oder logistische Kombinationen entwickeln, Marktchancen erspüren und dadurch das Wachstum der Wirtschaft vorantreiben. Ich spreche hier nicht nur von im rechtlichen Sinn Selbstständigen, Unternehmensgründern oder Freiberuflern, sondern auch über an innovativen Entwicklungen beteiligte Arbeitnehmer, über die viel zitierten „Unternehmer im Unternehmen“, die für den innovativen Prozess genauso unentbehrlich sind wie die Selbstständigen (das hatte Schumpeter unterschätzt). Für den Markterfolg der neuen Kombinationen ist nicht allein wichtig, dass die Erfindungen tatsächlich gemacht werden. Fast noch wichtiger ist ihre Kommunikation in Form von Ideen und „Visionen“; auch der viel kritisierte „symbolische“ oder „demonstrative“ Konsum sollte als ein Moment dieser symbolischen Inszenierungen begriffen werden (Deutschmann 2012). Das Aufstiegsmotiv wirkt somit als Motor für Erfindungen und Innovationen auf allen Ebenen des Wirtschaftssystems, vom einzelnen Arbeitsprozess bis hin zu ganzen Branchen und Volkswirtschaften; es scheint gerade in den unteren Mittelschichten besonders stark zu sein. Wiederum zeigt sich das nicht nur bei den häufig aus dem Kleinbürgertum stammenden selbstständigen Unternehmern (Beispiele: James Watt, Carl Siemens, Gottlieb Daimler, Henry Ford), sondern auch bei fachlich qualifizierten Arbeitern. Gerade in der sogenannten fordistischen, d. h. wohlfahrtsstaatlichen Epoche des Kapitalismus nach dem Zweiten Weltkrieg war das Aufstiegsmotiv auch bei Industriearbeitern ausgeprägt. Wenn man es selbst nicht schaffte, setzte man die Hoffnung wenigstens auf die eigenen Kinder. Viele verschuldeten sich, um den erhofften Erfolg gleichsam vorwegzunehmen, und setzen sich damit selbst unter Druck. Heute können wir solche Orientierungen bei manchen Einwanderern beobachten.

Der Kapitalismus - das ist damit auch gesagt - ist keine Veranstaltung zur freien Entwicklung menschlicher Kreativität, etwa wie die Kunst. Kreativität wird vielmehr durch soziale Ungleichheit, Konkurrenz und den daraus resultierenden Leistungsdruck mobilisiert. Dieser Druck gründet sich primär im Warencharakter des Arbeitsvermögens und wird sekundär durch die Kontrollmechanismen der Unternehmen verstärkt. Arbeitnehmer müssen kreativ sein, sie müssen vom Management gesteckte Ziele nicht nur erreichen, sondern übertreffen - obwohl es ohne ein Minimum an ,freiwilliger" Leistungsbereitschaft auch nicht geht. Mit dem Kontrollregime der Unternehmen bekommen wir eine weitere Ursache des kapitalistischen Wachstumszwangs in den Blick. In der Geschichte des Kapitalismus haben sich zwar die sozialen und kulturellen Formen dieses Kontrollregimes stark verändert: von den teils paternalistischen, teils quasi-militärischen Unternehmenshierarchien des 19. Jahrhunderts bis zu den heutigen, stark „subjektivierten“ Formen der Leistungskontrolle. Aber ihre Funktion ist gleich geblieben.

\section{Weitere Voraussetzungen: Bevölke- rungsentwicklung, institutionelle und infrastrukturelle Bedingungen}

Als zweites Ergebnis meiner Überlegungen ist somit festzuhalten: Es ist die teils durch das Aufstiegsmotiv, teils durch das Leistungsregime der Unternehmen getriebene „,außerordentliche“ Arbeitsbereitschaft der Vermögenslosen, die den innovativen Wachstumsprozess in Gang hält und damit rückwirkend die Verwertung des Kapitals der Vermögenden sicherstellt. Voraussetzung dafür ist eine Klassenstruktur, die die Ungleichheit von Kapital und Arbeit auf kollektiver Ebene festschreibt, auf individueller Ebene dagegen bis zu einem gewissen Grad offenhält und eine hinreichende Aufstiegsmotivation bei einer kritischen Masse der Vermögenslosen gewährleistet. Dieser hier nur stark stilisiert beschriebene Mechanismus ist schon komplex genug, und sein Funktionieren ist alles andere als selbstverständlich. Die Klassenstruktur kann z.B. aufgrund einer Verknüpfung mit 
traditionellen Sozialstrukturen „zu geschlossen“ sein und Aufstiege stark behindern. Sie kann aber auch im Gegenteil „zu offen“ oder egalitär sein, sodass keine starken Aufstiegsmotive mehr entstehen. Damit aber nicht genug: Damit der Wachstumsmechanismus funktionieren kann, müssen aus soziologischer Sicht noch eine Reihe weiterer Voraussetzungen gegeben sein.

An erster Stelle ist das Wachstum der Bevölkerung zu nennen. Die kapitalistische Wachstumsexplosion seit dem 19. Jahrhundert war nicht zufällig eine Bevölkerungsexplosion. Ein Wachstum der Bevölkerung führt in aller Regel auch zu einem Wachstum der wirtschaftlichen Produktion, allerdings nicht notwendigerweise auch zu einer Steigerung der Produktion pro Kopf, die ja mit dem Wachstumsbegriff eigentlich gemeint ist. Damit auch die Produktion pro Kopf steigen kann, muss es, wie gesagt, Innovationen und somit „Unternehmer“ geben. Auch hier kommt die Demografie ins Spiel, denn Unternehmer sind normalerweise junge Menschen, die ihr Leben noch vor sich haben und etwas aus ihm machen wollen. Unternehmer rekrutieren sich, wie die Sozialstatistik der Gründungen (Kelley et al. 2011, S. 32) zeigt, vorwiegend aus der Altersgruppe der jüngeren Erwachsenen. Die ideale Konstellation für kapitalistisches Wachstum ist eine stark wachsende, arme, zugleich nach Aufstieg strebende, vor allem aber: jugendliche Bevölkerung. Umgekehrt führt die Alterung der Bevölkerung an sich zu einer Verschlechterung der Wachstumschancen.

Eine weitere Voraussetzung für das Funktionieren des Wachstumsmechanismus sind die institutionellen und infrastrukturellen Rahmenbedingungen, deren Bedeutung vor allem in den neueren, institutionell orientierten Wachstumstheorien hervorgehoben wird. Die Konkurrenz um den sozialen Aufstieg bringt ja nicht schon von allein Innovationen und produktive wirtschaftliche Effekte hervor. Sie kann ebenso gut, wie die Erfahrungen in Schwellen- und Entwicklungsländern zeigen, mit illegalen Mitteln ausgetragen werden und die Form individueller oder organisierter Kriminalität annehmen. Die Ausrichtung des Aufstiegsstrebens auf den Erfolg nicht in lokalen Bandenkriegen, sondern am Markt, ist eine kulturell und sozial sehr voraussetzungsvolle Entwicklung. Vor allem auf Xavier Sala-i-Martin geht eine heute einflussreiche Unterscheidung von drei Bedingungskomplexen zurück, die sich zugleich als Indikatoren für das kapitalistische Entwicklungsniveau eines Landes interpretieren lassen (im Überblick Snowdon 2006). Der erste Komplex umfasst die „basic requirements“, also die grundlegenden institutionellen und infrastrukturellen Voraussetzungen kapitalistischer Entwicklung. Zu ihnen gehören ein funktionsfähiges Rechtssystem, das Eigentums- und persönliche Freiheitsrechte gewährleistet, eine elementare Bildung der Bevölkerung sowie eine hinreichende technische Infrastruktur im Verkehrswesen und in der Kommunikation. Der zweite Komplex wird mit dem Begriff „efficiency enhancers“ umschrieben. Er umfasst den Stand der sekundären schulischen Bildung der Bevölkerung, die Effizienz der Güter-, Arbeits- und Kapitalmärkte, ihre Transparenz und geografische Ausdehnung, schließlich das Niveau technologischen Wissens. Mit dem dritten, ,innovation and sophistication factors“ benannten Komplex werden die Potenziale einer Volkswirtschaft in Forschung, Entwicklung und industrieller Innovation umschrieben. Zusätzlich müsste man hier noch einen weiteren Faktor nennen, nämlich den modernen Sozial- und Wohlfahrtsstaat, der ja auch Aufstiegsmotive unterstützt, indem er Bildungsprozesse fördert und einen gewissen Schutz gegen die Entwertung erworbener Qualifikationen bietet.

\section{Industrie-, Schwellen- und Entwicklungsländer}

Die drei genannten Bedingungskomplexe beschreiben zugleich eine Hierarchie im kapitalistischen Entwicklungsgrad nationaler Volkswirtschaften. „Entwicklungsländer“ sind erst dabei, die grundlegenden Voraussetzungen kapitalistischer Innovation herzustellen, d.h. die Korruption zu bekämpfen, das Rechtssystem, das öffentliche Bildungswesen sowie eine leistungsfähige Infrastruktur für Verkehr und Kommunikation aufzubauen („factor driven economies“). In der zweiten Gruppe finden sich Länder, die in der Lage sind, Innovationen zu übernehmen, ohne sie selbst produzieren zu können, die Schwellenländer oder „efficiency driven economies“; weil die Übernahme von Innovationen kostengünstiger ist als ihre Produktion, weisen diese Länder oft besonders hohe Wachstumsraten auf. Schließlich gibt es die dritte Gruppe der „innovation driven economies“, d.h. die fortgeschrittenen Länder, die an vorderster Front des innovativen Prozesses stehen und die Technologieführerschaft auf den Märkten innehaben. Von ihnen hängt die Dynamik des globalen Wachstums letztlich ab.

Das skizzierte Modell leitet die Wachstumsdynamik einerseits aus sozialstrukturellen und demografischen, andererseits aus institutionellen und infrastrukturellen Bedingungen ab. Dabei spielt nicht nur eine Rolle, wie sich diese Bedingungen in der Perspektive des wissenschaftlichen Beobachters darstellen, sondern auch, wie sie durch die beteiligten Akteure selbst wahrgenommen werden. Dass ein von so komplexen Voraussetzungen abhängiger Prozess reibungslos funktioniert, ist höchst unwahrscheinlich. Es wäre naiv, hier eine eingebaute Tendenz zum „Gleichgewicht“ anzunehmen. Die krisenhafte Realität kapitalistischer Entwicklung zeigt, dass eine durchweg wachstumsförderliche Bedingungskonstellation nicht als Regel, sondern als historische Ausnahme zu betrachten ist. Es gibt keinerlei Garantie dafür, dass die genannten Bedingungskomplexe sich gegenseitig stützen oder verstärken, im Gegenteil scheinen sie eher negativ miteinander verknüpft zu sein. Dort nämlich, wo die demografischen und auch die sozialstrukturellen Bedingungen eine günstige Prognose für das Wachstum nahelegen, sind die infrastrukturellen und institutionellen Bedingungen oft eher unterentwickelt. Umgekehrt weisen avancierte Volkswirt- 
schaften mit hervorragend entwickelter institutioneller und materieller Infrastruktur unter sozialstrukturellen und demografischen Aspekten oft eher ungünstige Voraussetzungen für wirtschaftliches Wachstum auf.

Der Vergleich zwischen der Gruppe der Industrieländer einerseits, der Schwellen- und Entwicklungsländer andererseits kann das verdeutlichen. Hoch entwickelte Industrieländer wie die Kernländer der EU, die USA und Japan sind durch einen hohen sekundären und tertiären Bildungsstand der Bevölkerung und eine vielfach gut entwickelte Forschungsinfrastruktur charakterisiert und weisen einen hohen Grad an Rechtssicherheit auf - Bedingungen, die in den Schwellen- und vor allem den Entwicklungsländern vielfach fehlen oder nur unzureichend gewährleistet sind. Hinsichtlich der demografischen und sozialstrukturellen Gegebenheiten jedoch sind die Wachstumsbedingungen in den Entwicklungs- und Schwellenländern, mit ihrer oft noch stark anwachsenden Bevölkerung und ihrer hohen, freilich oft durch Not motivierten unternehmerischen Gründungsaktivität (Kelley at al. 2011), deutlich besser. In den reifen Industrieländern dagegen altert die Bevölkerung, stagniert bzw. geht in manchen Ländern (z. B. Japan) sogar stark zurück. Die Ungleichheiten in der Verteilung von Einkommen und Vermögen haben, auch aufgrund der Vererbung von Bildungs- und Vermögensdifferenzen, stark zugenommen (Piketty 2014). Zugleich haben sich die Aufstiegschancen für Personen mit niedrigem Einkommen und geringer Bildung stark verschlechtert. Außerdem haben sich die durch einen eher saturierten sozialen Habitus geprägten oberen Mittelschichten vergrößert, sodass das soziale Reservoir für potenzielle unternehmerische Aufsteiger aus den unteren Mittelschichten kleiner geworden ist. Das wirkt sich auch negativ auf die unternehmerische Gründungsaktivität aus: Unternehmensgründungen werden in diesen Ländern riskanter, schwieriger und weniger attraktiv (Kelley et al. 2011; für Deutschland: ZEW 2014). Hinzu kommt, dass neue große Innovationen, die Gründungen motivieren könnten, gegenwärtig kaum in Sicht sind (ob die Energiewende eine solche Rolle in absehbarer Zeit spielen kann, erscheint mir zweifelhaft). Das von mir oben skizzierte Idealszenario für eine positive Wachstumsdynamik ist in den entwickelten Ländern gerade aufgrund der erfolgreichen Entwicklung in der zweiten Hälfte des 20. Jahrhunderts (die vielen Menschen den Aufstieg ermöglicht hat und die Mittelschichten hat anwachsen lassen) nicht mehr gegeben (ausführlicher: Deutschmann 2008, 2009).

\section{Schlussfolgerungen}

Ich komme zur Ausgangsfrage zurück: Ist eine Ökonomie ohne Wachstumszwang ein Wunschtraum? Mein Fazit ist, dass die Frage für die entwickelten Länder (Westeuropa, Nordamerika, Teile Ostasiens) anders zu beantworten ist als für die Schwellen- und Entwicklungsländer. Das Problem in den entwickelten Ländern ist, dass die Wachstumszwänge mit den kapitalistischen Eigentumsverhältnissen erhalten bleiben, das Wachstum selbst jedoch trotz der hoch entwickelten materiellen und institutionellen Infrastruktur aufgrund der sozialstrukturellen Bedingungskonstellation zurückgeht und zukünftig ganz ausbleiben könnte. Weil „Unternehmertum“ hier immer häufiger auf eine prekäre Existenz hinausläuft (Bührmann/Pongratz 2010), sind immer weniger Menschen bereit, die Risiken einer unternehmerischen Existenz freiwillig zu übernehmen. Darüber hinaus schrumpft mit der Alterung der Bevölkerung auch das soziale Reservoir unternehmerischer Aufsteiger. Während die Nachfrage nach Risikokapital faktisch abnimmt, nehmen die anlagesuchenden Finanzvermögen der oberen Schichten zu. Die Folge ist ein struktureller Angebotsüberhang an den Kapitalmärkten, der zur Bildung von Finanzblasen führt und die wirtschaftliche Dynamik lähmt (Deutschmann 2008). Die Frage, ob weiteres Wachstum noch „wünschenswert“ ist oder ob wir es uns aus ökologischen Gründen noch „leisten“ können, wird sich hier also gar nicht stellen, denn die wirtschaftliche Dynamik wird so oder so zurückgehen und die Gefahr eines dauernden ökonomisch-sozialen Niedergangs heraufbeschwören. Eine kapitalistische Wirtschaft, die nicht mehr wächst, kann auch nicht mehr einfach auf dem gleichen Niveau weitermachen, sondern entwickelt eine negative, auf Rückgang und Schrumpfung gerichtete Dynamik. Das ist kein Wunschtraum, sondern ein Albtraum.

Die zentrale Frage wird sein, wie man diese negative Dynamik aufhalten und die wirtschaftliche Produktion auch dann weiterführen kann, wenn sie keinen oder nur noch geringen Gewinn abwirft. Damit ist die Eigentumsfrage aufgeworfen, denn der Wachstums- und Gewinnzwang wurzelt, wie ich ausgeführt hatte, in der Trennung von Arbeitskraft und Produktionsmitteln. Der Wachstumszwang - auch da hatte Marx Recht - kann letztlich nur durch eine Veränderung der Eigentumsformen überwunden werden. Man wird die Trennung zwischen Arbeit und Kapital nicht über Nacht rückgängig machen können. Aber es empfiehlt sich, über eigentumsrechtliche Veränderungen nachzudenken, um die gesellschaftlich zunehmend negativen Folgen dieser Trennung zu begrenzen. Infrage kommen eine Verstaatlichung zu groß gewordener Banken und Unternehmen, der Ausbau des öffentlichen Sektors, insbesondere aber die Förderung genossenschaftlichen Eigentums (Schmale 2013) sowie des von Stiftungen und Zuwendungen getragenen „dritten Sektors". Auf Kapital wird man bis auf Weiteres nicht verzichten können, gefordert ist jedoch nur noch „geduldiges“, nicht an schneller und hoher Rendite orientiertes Kapital. Das wird keineswegs das Ende des „Unternehmers“ bedeuten, im Gegenteil: Unternehmertum im sozialen und kommunalen Bereich wird immer mehr gefragt sein (Hulgard 2011). Auch der schon heute in der Managementliteratur gefeierte „Arbeitskraft-Unternehmer“(Voß/Pongratz 1998) wird weiterhin eine wichtige Rolle spielen: Gerade in fortge- 
schrittenen Industriezweigen wie der IT-Branche besteht das wichtigste „Kapital“ der Unternehmen heute ohnehin in dem Wissen und den Kompetenzen der Mitarbeiter. Es ist auch nichts dagegen einzuwenden, dass die Gewinne aus erfolgreichen Gründungen in angemessenem Ausmaß auch den Unternehmern selbst zufließen. Der Rentier dagegen wird zu einer anachronistischen Figur. Gegen Sparen und den späteren Verbrauch des Ersparten ist zwar nichts einzuwenden. Aber von der Vorstellung, dass gespartes Geld nur „angelegt“ werden muss und sich dann von allein vermehrt, werden wir uns verabschieden müssen. Auch in fernerer Zukunft ist in den entwickelten Ländern kaum mehr damit zu rechnen, dass Kapitalanlagen - von spekulativen und entsprechend risikobehafteten Geschäften abgesehen - noch eine nennenswerte Rendite abwerfen. Die von Keynes schon vor 80 Jahren erhobene Forderung nach einer „Euthanasie des Rentiers“ gewinnt mehr denn je Aktualität. Der Finanzsektor wird drastisch schrumpfen, und das wird eine weitreichende Umstellung des Lebensstils auch der vermögenden Mittelschichten bedeuten.

Anders die Situation in den Entwicklungsländern und in Schwellenländern wie Indien, Brasilien, Russland oder Südafrika, in denen die institutionellen und infrastrukturellen Rahmenbedingungen in den letzten Jahrzehnten deutlich verbessert worden sind. Trotz der in diesen Ländern vielfach noch immer herrschenden extremen sozialen Ungleichheiten und der verbreiteten Korruption scheinen die oben beschriebenen Wachstumsmotoren hier besser zu funktionieren, oder sie beginnen erst zu funktionieren, wie anscheinend in Afrika. Vor allem aber wächst die Bevölkerung. Den Millionen von Menschen in diesen Ländern, die auf eine Verbesserung ihrer Lage hoffen, wird die Kritik am „Wachstumszwang“ weltfremd erscheinen. Aber gerade deshalb wird sich die ökologische Frage hier in extremer Form stellen. Den in den Entwicklungsländern und teils auch noch in den Schwellenländern anstehenden Wachstumsschub aus Gründen des Umweltschutzes zu unterbinden, wäre weder möglich noch wünschenswert. Der Zielkonflikt zwischen den sozialen und den ökologischen Aspekten des Wachstums wird sich also zuspitzen, ohne dass gegenwärtig eine Lösung in Sicht wäre. Aber in manchen Schwellenländern, insbesondere in China nähert sich die Konstellation trotz aktuell noch hoher Wachstumsraten immer mehr jener der entwickelten Ökonomien an. Auch die heutigen Schwellenländer werden es in nicht allzu ferner Zukunft mit den Problemen der „reifen“ Länder zu tun bekommen, von denen die wirtschaftliche Gesamtdynamik ja letztlich abhängt. Nicht heute, wohl aber auf mittlere Sicht ist damit zu rechnen, dass die Überwindung des Wachstumszwangs zu einer globalen Herausforderung wird.

\section{LITERATUR}

Bührmann, A./Pongratz, H. J. (2010): Prekäres Unternehmertum. Unsicherheiten von selbstständiger Erwerbstätigkeit und Unternehmensgründung, Wiesbaden Deutschmann, C. (2008): Die Finanzmärkte und die Mittelschichten: der kollektive Buddenbrooks-Effekt, in: Leviathan 36 (4), S. 501-517

Deutschmann, C. (2009): Soziologie kapitalistischer Dynamik: Max-Planck-Institut für Gesellschaftsforschung, MPIfG Working Paper 09/5, Köln

Deutschmann, C. (2011): A pragmatist theory of capitalism, in: Socio-Economic Review 9 (1), S. 83-106

Deutschmann, C. (2012): Die Dynamik des Konsums und die moralische Integration moderner Gesellschaften, in: Leviathan 40 (4), S. 517-535

Esser, H. (1993): Soziologie. Allgemeine Grundlagen, Frankfurt a. M.

Freeman, C./Louca, F. (2001): As time goes by. From the industrial revolutions to the information revolution, Oxford

Gross, P. (1994): Die Multioptionsgesellschaft, Frankfurt a. M.

Hulgard, L. (2011): Social economy and social enterprise: an emergent alternative to mainstream market economy, in: China Journal of Social Work 4 (3), S. 201-215
Kelley, D./Bosma, N./Amorós, J. E. (2011): Global entrepreneurship monitor, 2010 global report, Babson College, Babson Park MA.

Maddison, A. (2001): The world economy. A millenial perspective, Paris

Marx, K. (1988): Das Kapital. Kritik der Politischen Ökonomie, Bd. 1, MEWGesamtausgabe, Bd. 23, Berlin

Merton, R. K. (1968): Social theory and social structure, enlarged edition, New York

Miegel, M. (2010): Exit. Wohlstand ohne Wachstum, Berlin

Piketty, T. (2014): Capital in the twenty-first century, Cambridge/Mass.

Schmale, I. (2013): Genossenschaften: eine traditionsreiche und zukunftsfähige Unternehmensform, in: Zeitschrift für Sozialökonomie 50 (176/177), S. 23-28

Snowdon, B. (2006): The enduring elixir of economic growth. Xavier Sala-i-Martin on the wealth and poverty of nations, in: World Economics 7 (1), S. 73-130 Voß, G./Pongratz, H. G. (1998): Der Arbeitskraftunternehmer. Eine neue Grundform der „Ware Arbeitskraft”?, in: Kölner Zeitschrift für Soziologie und Sozialpsychologie 50 (1), S. 131-158

Wallerstein, I. (1979): The capitalist world economy, Cambridge Zentrum für Europäische Wirtschaftsforschung (ZEW) (2014):

Mannheimer Unternehmenspanel: Junge Unternehmen: Fakten, Analysen, Perspektiven (1), Mannheim

\section{AUTOR}

CHRISTOPH DEUTSCHMANN, Dr. habil., Professor für Soziologie an der Universität Tübingen (i.R.). Arbeitsschwerpunkte: Arbeits- und Wirtschaftssoziologie.

christoph.deutschmann@uni-tuebingen.de 